\title{
Field-dependence and form discrimination in females
}

\author{
GLEN M. VAUGHT AND BARBARA AUGUSTSON
}

ALBION COLLEGE

Forty-two females were divided into field-independent, medium, and field-dependent groups and administered a form discrimination task. Contrary to previous findings, both the field-dependent and the medium group made fewer errors than the field-independent group in passive touch.

Recent studies have shown that field-dependence and/or the global-articulated dimension of cognitive functioning cuts across sense modalities. Support for this position has come from a number of studies employing a variety of measures as well as subjects. For example, Axelrod \& Cohen (1961) reported a correlation of .78 between scores on a visual embedded-figures test and performance in a form discrimination task for adult males. Similar relationships have been cited by Witkin, Birnbaum, Lomonaco, Lehr, \& Herman (1965) in both sighted and totally blind boys and girls. Vaught \& Ellinger (1966), using college students of both sexes, reported a significant correlation of -.50 between Rod-and-Frame Test (RFT) scores and form discrimination; i.e., the fewer the deviations from true vertical in the RFT, the greater the accuracy in form discrimination.

With the possible exception of the Vaught \& Ellinger (1966) study, little or no research has been done in which field-dependence has been related to active and passive touch, although it has been well established that people differ in ability to discriminate among random shapes under conditions of active-passive touch (Gibson, 1962). The active-passive dichotomy in form discrimination provides a means of acquiring a more complete knowledge of the manner in which field-dependence affects form discrimination. Such an approach is thought to lend a certain degree of specificity to the predictive value of the field-dependence dimension.

The present study sought to determine the manner in which females, who vary along the field-dependence dimension, perform in both active and passive touch. The use of females was in response to the finding that the strongest correlation between field-dependence and form discrimination occurred in active touch for females (Vaught \& Ellinger, 1966).

\section{Method}

The Ss were 42 female introductory psychology students from Albion College, Albion, Michigan, selected from a pool of $\mathrm{Ss}$ who had previously taken the RFT. The Ss were divided into three subgroups; field-independent (FI), Medium (Med.), and field-dependent (FD), with 1- Ss per group. Table 1 shows the mean RFT score for each identity group.
The materials and procedure were essentially the same as those reported by Vaught \& Ellinger (1966) with some slight modifications. The form discrimination task consisted of six Masonite forms patterned ufter those employed by Gaydos (1956). Each form was reproduced in identical shape and size with black ink on white posterboard, and was numbered for purposes of identification. The forms were prezented in an up-side-down cardboard box that was draped on one side by a black cloth. All stimuli were shielded from the Ss' sight in this manner.

Each condition in which an $\mathrm{S}$ performed was explained, but no knowledge of results nor any information is to the number of trials to be run was given. The six forms were randomly presented to the $S$ until each form had been presented four times under each of two conditions, an active and a passive condition. Every $S$, therefore, ran a total of 48 trials with each trial lasting approximately 5 sec.

In the active condition, the $S$ was asked to place her dominant hand inside the box palm down and to hold it above the bottom of the box. A stimulus form was drawn from the container and laid beneath the S's hand. On the command "go," the S picked up the form, felt it, and then visually identified, by number, the figure on the chart that she thought was identical to the form in her hand. At the end of $5 \mathrm{sec}$., the $S$ dropped the form on the command "drop" but was still allowed to respond if she had not done so already.

In the passive condition, the $S$ was again instructed to put her hand inside the box, but with her palm up. Each form was then placed in the S's hand simultaneously with the "go" command and the trial then continued as described earlier. Unlike the previous study by Vaught \& Ellinger (1966), the $S$ was not allowed to "grasp" the form. It was assumed that this procedure increased the difficulty of the task by cutting down on the amount of sensory experience for each $\mathrm{S}$.

Presentation of the stimulus for each trial was randomized by arbitrarily picking a form out of a box containing all six forms. The trials were balanced for each $\mathrm{S}$ by presenting 12 trials under one

Table 1. Mean Error Scores for all Groups

\begin{tabular}{llrrr} 
& N & RFT & $\begin{array}{c}\text { Active } \\
\text { Touch }\end{array}$ & $\begin{array}{c}\text { Possive } \\
\text { Touch }\end{array}$ \\
\hline FI & 14 & 1.09 & 1.64 & 8.07 \\
Med. & 14 & 10.45 & 1.14 & 6.07 \\
FD & 14 & 30.62 & 1.14 & 5.57 \\
\hline
\end{tabular}


condition, 24 under the other, and 12 more under the first, in an ABBA sequence. The Ss themselves were balanced by alternating the ABBA sequence with a $B A A B$ sequence of trials for every other $S$. In this arrangement one $S$ would begin and end with 12 active trials in the ABBA sequence while the next $S$ would begin and end with 12 passive trials in a BAAB sequence. These precautions were taken to balance learning by the Ss and to control for any influence one particular sequence might have on another.

\section{Results and Discussion}

A Winer (1962) repeated measures analysis of variance was performed on the form discrimination error scores. The three groups (FI, Med, and FD) did not differ significantly in the number of errors for active and passive touch combined $(F=3.13 ; 3.25$ needed for .05 level). While the accepted level of significance was not reached, it is nevertheless important to note the trend toward a greater number of errors in form discrimination as field-independence increased. The direction of this trend was not in keeping with the overall correlation (males and females combined) between RFT scores and form discrimination reported by Vaught \& Ellinger (1966).

A highly significant difference $(p<.001)$ was found between the active touch mean of 18.33 and the passive touch mean of 92.00. All groups made fewer errors under conditions of active touch. The Newman-Keuls procedure was used to compare the individual group means shown in Table 1 . The three groups did not differ in terms of the number of errors made in active touch. Again, this finding failed to support the Vaught \& Ellinger (1966) study in which females made fewer errors in form discrimination in the active touch condition as field-independence increased. The passive touch means, however, did differ significantly, i.e., both the FD and the Med. group made fewer errors than the FI group $(p<.05)$.
The present results are difficult to explain in view of their departure from previous work. On hypothesis that would explain the present findings concerns the nature of the requirements in both the RFT and form discrimination. It could be that the FI females who use themselves as a frame of reference in the RFT, when confronted with the discrimination task, continue to rely on themselves, and in doing so lose a good deal of the available "sensory information" needed to make a valid judgment. Whereas, the FD female uses the frame as a reference point in RFT and continues to rely on external information in the discrimination task. The FD females, then, would be in a position to acquire much more sensory experience than the FI females. Additional support for this position comes from the present finding that when the amount of sensory experience is decreased, as in passive touch, FI females made more errors than either the Med. group or the FD group. It is obvious that much work needs to be done in this general area. The authors have research under way in order to determine whether males behave in a similar way.

\section{References}

Axelrod, S., \& Cohen, L. D. Senescence and embedded-figures performance in vision and touch. Percept. mot. Skills, 1961, $12,283-288$.

Gaydos, H. Intersensory transfer in the discrimination for form. Amer. J. Psychol., 1956, 69, 107-110.

Gibson, J. J. Observations in active touch. Psychol. Rev., 1962, $69,477-490$.

Vaught, G. M., \& Ellinger, J. Field-dependence and form discrimination. Psychon. Sci., 1966, 6, 357-358.

Winer, B. J. Statistical principles in experimental design. New York: McGraw-Hill, 1962.

Witkin, H. A., Bimbaum, Judith, Lomonaco, S., Lehr, Suzanne, \& Herman, Judith. Cognitive patterning in congenitally totally blind children. Paper presented at the 1965 annual meeting of the American Psychological Association. 